\title{
Multifocal glioblastoma-two case reports and literature review
}

\author{
Zuo-Xin Zhang ${ }^{1}$, Ju-Xiang Chen ${ }^{2}$, Bao-Zhong Shi ${ }^{3}$, Guang-Hui Li ${ }^{4}$, Yao Li ${ }^{1}$, Yan Xiang ${ }^{1}$, Xun Qin ${ }^{1}$, Lin Yang ${ }^{1}$ and \\ Sheng-Qing LV ${ }^{1 *}$
}

\begin{abstract}
Background: Multifocal glioblastoma is a rare type of glioblastoma with worse prognosis. In this article, we aimed to report two cases of classical multifocal glioblastoma.

Case presentation: In case 1, a 47-year-old male presented with dizziness, and once had a sudden loss of consciousness accompanied by convulsion of limbs. Contrast-enhanced MRI showed multiple lesions with heterogeneously ring-enhanced characters in the left hemisphere, diagnosed as multifocal glioblastoma. He underwent a craniotomy of all lesions, concurrent radiotherapy and chemotherapy as well as additional chemotherapy of temozolomide. After 2 cycles, repeat MRI showed that the new lesions already occurred and progressed. Eventually, he abandoned the chemotherapy after the 2 cycles and died 1 year later. In case 2, a 71year-old male presented with a history of headache, left limb weakness, and numbness. Discontinuous convulsion of limbs once occurred. Contrast-enhanced MRI showed multiple lesions located in the right hemisphere, diagnosed as multifocal glioblastoma. He underwent a right frontoparietal craniotomy of the main lesion. Hemorrhage of the residual tumor and pulmonary artery embolism occurred synchronously. Eventually, his family decided not to pursue any further treatment and opted for hospice care and he passed away within 11 days of surgery.
\end{abstract}

Conclusions: We reported two cases of typical multifocal glioblastoma. Valid diagnosis is crucial; then, resection of multiple lesions and canonical radio-chemotherapy probably bring survival benefits.

Keywords: Multifocal glioblastoma, Surgical resection, Radio-chemotherapy

\section{Background}

Glioblastoma multiforme (GBM) is the most common primary malignancy in central nervous system with high aggressiveness and extremely poor prognosis [1]. Although surgical resection with maximum safe range and additional radio-chemotherapy remains the standard treatment of GBM, the median survival of GBM is approximately less than 15 months [2]. Nowadays, the brain metastases, such as lung cancer, which is the most

\footnotetext{
*Correspondence: Ivsq0518@hotmail.com

'Department of Neurosurgery, Xinqiao Hospital, Third Military Medical University, No.183 Xinqiao Street, Shapingba District, Chongqing City 400037, People's Republic of China

Full list of author information is available at the end of the article
}

common source [3], are becoming more prevalent and common than primary brain cancer [4]. And the characteristic radiologic imaging feature of brain metastases, which is similar with the other diseases of intracranial multiple lesions, always results in misdiagnosis in the clinical management.

Multifocal glioblastoma is a rare type of glioblastoma multiforme and it associates with worse prognosis compared with solitary ones [5]. It manifests as multiple distinct lesions simultaneously, exhibiting a clear pathway of spread lesions [6] and there is a presumed microscopic connection among them [7]. It possesses the similar properties of radiologic imaging and can be sometimes misdiagnosed as brain metastases, which

(C) The Author(s). 2021 Open Access This article is licensed under a Creative Commons Attribution 4.0 International License, which permits use, sharing, adaptation, distribution and reproduction in any medium or format, as long as you give appropriate credit to the original author(s) and the source, provide a link to the Creative Commons licence, and indicate if changes were made. The images or other third party material in this article are included in the article's Creative Commons licence, unless indicated otherwise in a credit line to the material. If material is not included in the article's Creative Commons licence and your intended use is not permitted by statutory regulation or exceeds the permitted use, you will need to obtain permission directly from the copyright holder. To view a copy of this licence, visit http://creativecommons.org/licenses/by/4.0/. The Creative Commons Public Domain Dedication waiver (http://creativecommons.org/publicdomain/zero/1.0/) applies to the data made available in this article, unless otherwise stated in a credit line to the data. 
creates enormous challenges in the subsequent management.

Here, we present two cases of typical multifocal glioblastoma and we also have reviewed related literatures, and the aim of this report is to discuss the diagnosis and treatment of this peculiar malignancy.

\section{Case presentation}

\section{Case 1}

A 47-year-old male presented with dizziness for 10 days, and he also complained that he once had a sudden loss of consciousness accompanied by convulsion of his limbs which lasted for $3 \mathrm{~min}$ at the this duration. Syndromes of headache, vomiting, and hypoplasia were not described. Mild cognitive impairment and weakness of the right side were detected at admission.

Computed tomography (CT) of the brain were performed, which manifested multiple intracranial lesions synchronously. Positron emission tomography-computed tomography (PET-CT) revealed no abnormal neoplasms in the other tissues of the body. The magnetic resonance imaging (MRI) revealed 4 occupying lesions with surrounding edema in the left frontal and parietal lobes. Deep-seated lesions invaded to the parenchyma adjacent to the left ventricle, contributing to the mass effect and middle line shift. The whole masses presented with a heterogeneously ring-enhanced character with necrosis and cystic changes after gadolinium (Gd) administration (Fig. 1a-i).

The patient underwent a left frontoparietal craniotomy of 3 lesions with the guidance of intraoperative navigation and sodium fluorescence-guided technique. Lesions were seen with grey appearance, obscure boundary, and rich blood supply and removed for decompression of the intracranial pressure. We successfully resected the masses micro-surgically for additional biopsy. Then, the gross total resection of these masses around their approximate boundary was achieved. We used the same way to resect the last lesion in occipital lobe under prone position.

Histopathological examination revealed that these tumor cells possessed increased necrosis, and significant heterogeneity of nucleus (Fig. 1j, k). Immunohistochemical staining demonstrated that these lesions shared the similar characters of intensity of the biomarkers such as glial fibrillary acidic protein (GFAP) and Olig-2. The abnormal expression of Ki-67 (20\%) revealed the potential proliferation activity of the tumor cells which indicated poor prognosis and high incidence of recurrence. Both the lesions showed the amplification of epidermal growth factor receptor (EGFR), which indicated the high aggressiveness. Pathological results were compatible with a diagnosis of multifocal glioblastoma. We also performed the whole genome sequencing (WGS) of the four lesions. The WGS results revealed that four samples had TERT promoter mutation C228T (chr5:1,295,228:C $>$ T), but none of IDH1/2 mutation, ATRX mutation, and EGFRvIII were detected.

The patient presented with no other abnormal neurological signs and his right limb's strength had improved gradually after operation. There was no recurrence of his epilepsy at this duration. After 5 weeks, a follow-up MRI
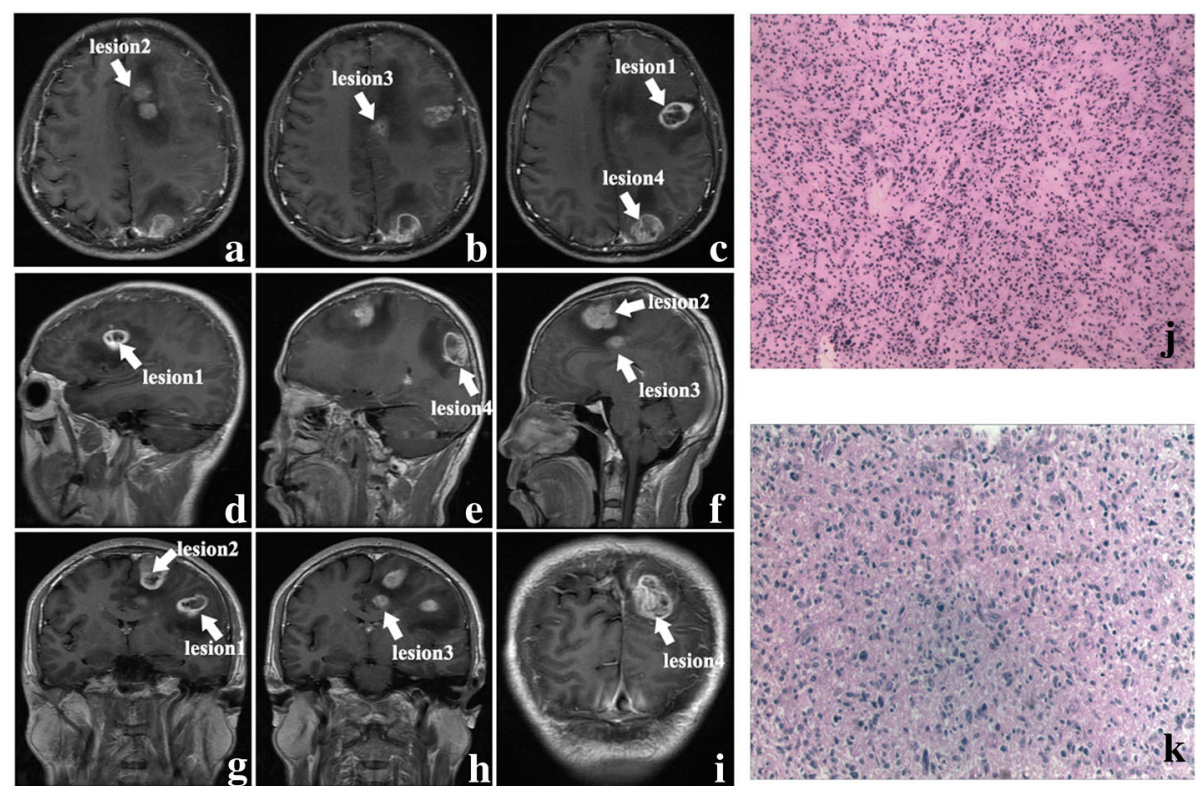

Fig. 1 The axial, sagittal, and coronal contrast-enhanced MRI images (a-i) (arrows represent the lesions). The hematoxylin-eosin (HE) staining of intraoperative frozen histologic section and paraffin section $(\mathbf{j}, \mathbf{k}, \times 100)$ 
was performed before radiotherapy and revealed that local tumor control was achieved and the initial resection cavities were seen; however, two new lesions occurred adjacent to the primary lesion areas and exhibited intense enhancement; nevertheless, remarkable mass effect and middle line shift were not observed either (Fig. 2a-i). Concurrent radiotherapy and chemotherapy were then administered to the patient.

Intensity-modulated radiotherapy (IMRT) targeted these lesions and areas of enhancement. A dosage of $60.0 \mathrm{~Gy}$ in 30 fractions were performed and constantly orally administered temozolomide (TMZ), with a dosage of $120 \mathrm{mg}$ ( $75 \mathrm{mg} / \mathrm{m} 2 /$ day) per day for 46 days were also performed to achieve the concurrent radio-chemotherapy. Sideeffects and toxicity such as liver and hematopoietic dysfunction did not occur.

The following adjuvant chemotherapy of temozolomide with $240 \mathrm{mg}(150 \mathrm{mg} / \mathrm{m} 2 /$ day $)$ for 5 days every 28 days was then administered to the patient. Hematologic and gastrointestinal toxicity was examined every cycle and no significant abnormality was detected. We also monitored the functions of liver and kidney constantly. Dysfunctions of the organs and anaphylaxis were also not seen. After 2 cycles, the repeat MRI revealed that the former two new lesions enlarged distinctly, manifesting ring-enhanced character, and two more new lesions had emerged again, infiltrating the white matter tract of ventricles and parenchyma (Fig. 3a-i). Eventually, the patient abandoned the chemotherapy after the 2 cycles due to his low quality of life. The patient died 1 year later.

\section{Case 2}

A 71-year-old male presented with a history of headache, left limb weakness, and numbness for 1 month. He also described that discontinuous convulsion of the limbs once occurred when he asked for medical service at this duration. Syndrome of vomiting, hypopsia, and cognitive impairment were not detected.

Gadolinium-enhanced MRI was performed, demonstrating 4 well-defined, highly enhanced lesions located in the right frontal and parietal lobes which were suspicious for metastatic lesions, and one of them was adjacent to the right lateral ventricle (Fig. 4a-f). PETCT was also performed and showed hypometabolic foci of FDG corresponding to enhancing lesions seen on the MRI, but the chest, abdomen, and pelvis were negative for any primary lesion.

To achieve the goals of safe resection and obtain the mass for histopathologic diagnosis, the patient underwent a right frontoparietal craniotomy of the main lesion near the surface of parenchyma with the guidance of intraoperative navigation and intravenous
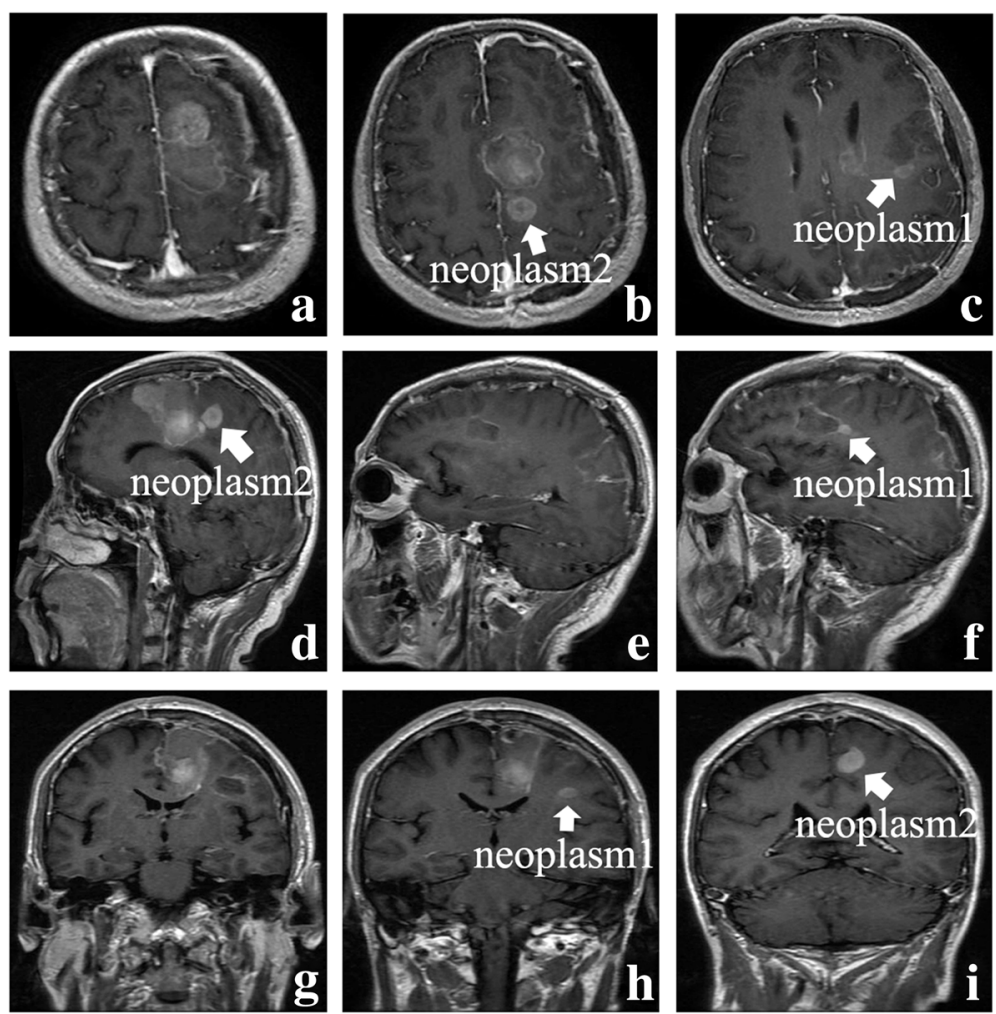

Fig. 2 The axial, sagittal, and coronal contrast-enhanced MRI images after 5 weeks of surgery (a-i). Two new lesions occurred adjacent to the primary lesion area (arrows represent the neoplasms) 

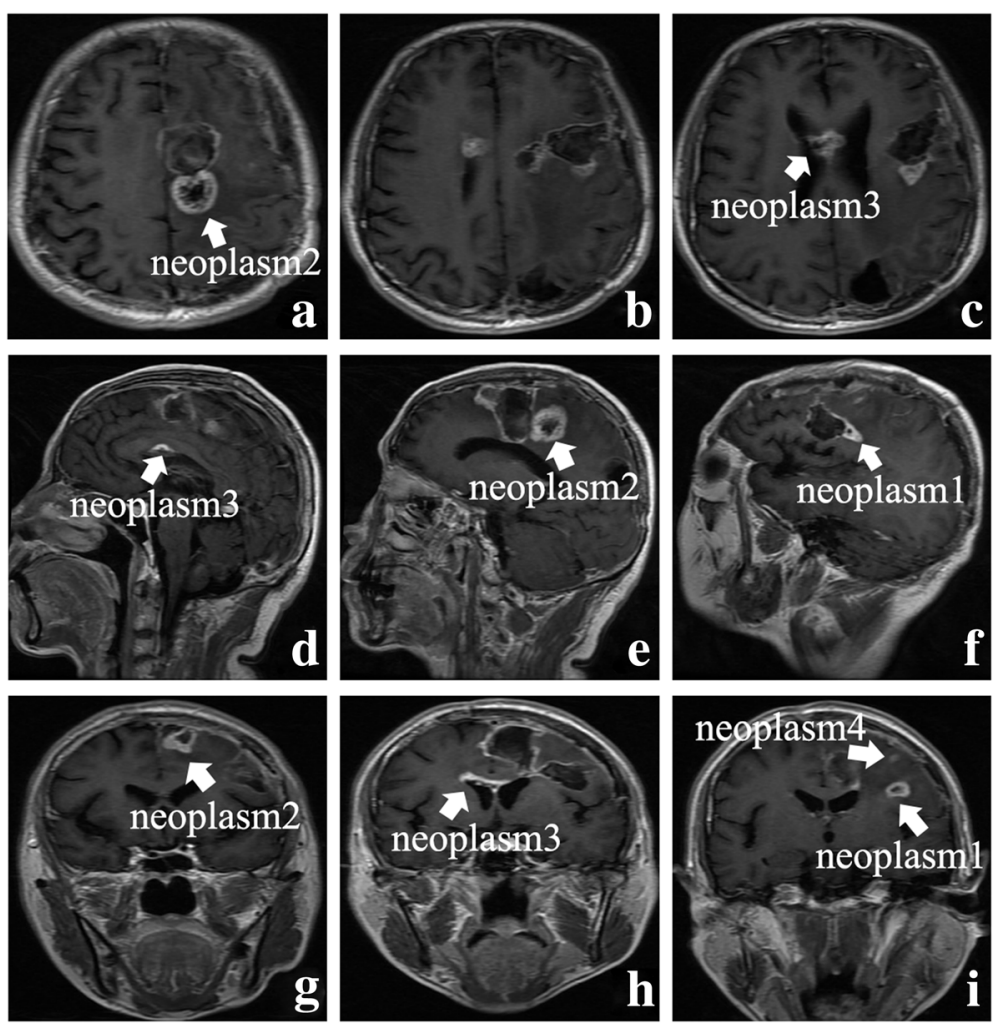

Fig. 3 The axial, sagittal, and coronal contrast-enhanced MRI images after 2 cycles of chemotherapy (a-i). Former two new lesions enlarged distinctly; two more new lesions had emerged again (arrows represent the neoplasms)
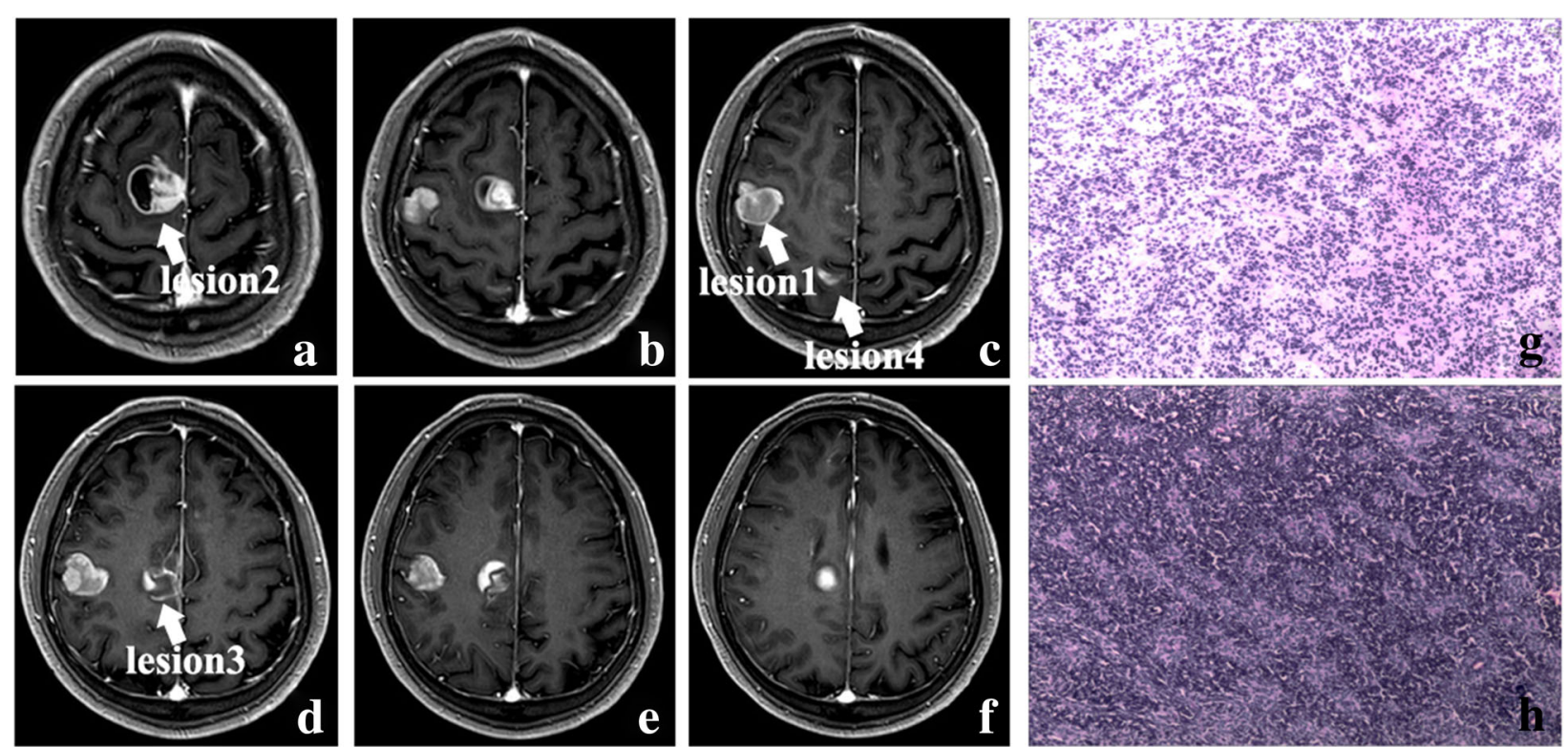

Fig. 4. The axial contrast-enhanced MRI images (a-f) (arrows represent the lesions). The hematoxylin-eosin (HE) staining of intraoperative frozen histologic section and paraffin section $(\mathbf{g}, \mathbf{h}, \times 100)$ 
fluorescein. The mass was seen with grey and white appearance, obscure boundary, and moderate blood supply. The gross total resection of the mass around its approximate boundary was also achieved (Fig. 5af) and no significant postoperative abnormal neurological signs were detected.

Histopathological examination revealed infiltrating highly proliferative cells with nuclear atypia which fulfilled the criteria of malignant glioma (Fig. $4 \mathbf{g}$, h). Immunohistochemical staining demonstrated the highly expressed Ki-67 index (30\%), positive GFAP, Olig-2, CD34, CD99, P53, and ATRX whereas, negative IDH1, NeuN, and Vimentin. The final results were also compatible with a diagnosis of multifocal glioblastoma.

The patient suddenly presented a sign of dyspnea at the 10th day after operation, followed with decreased conscious level and blood-oxygen saturation. The emergent CT showed hemorrhage of the residual tumor located in the right parietal lobe closed to the mid-line (Fig. 5g); meanwhile, the CT angiography of pulmonary artery showed a sign of pulmonary artery embolism (Fig. 5h). Conservative treatment was implemented in consideration of the condition of the patient; eventually, the patient's family decided not to pursue any further treatment and opted for hospice care and the patient passed away within 11 days of surgery.

\section{Discussion}

Newly diagnosed multiple glioblastoma can be termed as multifocal or multicentric [8]; generally, multifocality is diagnosed when connections between lesions present and tumors always disseminate via parenchymal routes [9]. Previous studies reported that incidence of multifocal glioblastoma is ranging from 0.5 to $20 \%$ [10, 11], and patients of multifocal glioblastoma demonstrated worse prognosis. Patil et al. reported that a significantly shorter median overall survival of 6 months was observed in multifocal glioblastoma compared with that of solitary glioblastoma, of which was 11 months, and 2year survival rates of multifocal glioblastoma were significantly lower than that of solitary glioblastoma [12]. Lasocki et al. reported that patients of multifocal glioblastoma generally live a median survival of 6-8 months from diagnosis [13]. The hypothesized analysis of this dilemma may include higher burden of disease, genetically more aggressive phenotype and inability for gross total resection of the multiple lesions [6]. In our case 1, although the patient received gross total resection of the multiple lesions and additional radio-chemotherapy, new lesions emerged and enlarged shortly after and the patient died 1 year later, demonstrating their strong aggressiveness.

Multiple intracranial lesion imaging characters represent a diagnostic dilemma in that multiple ringenhancing lesions usually were diagnosed as metastatic

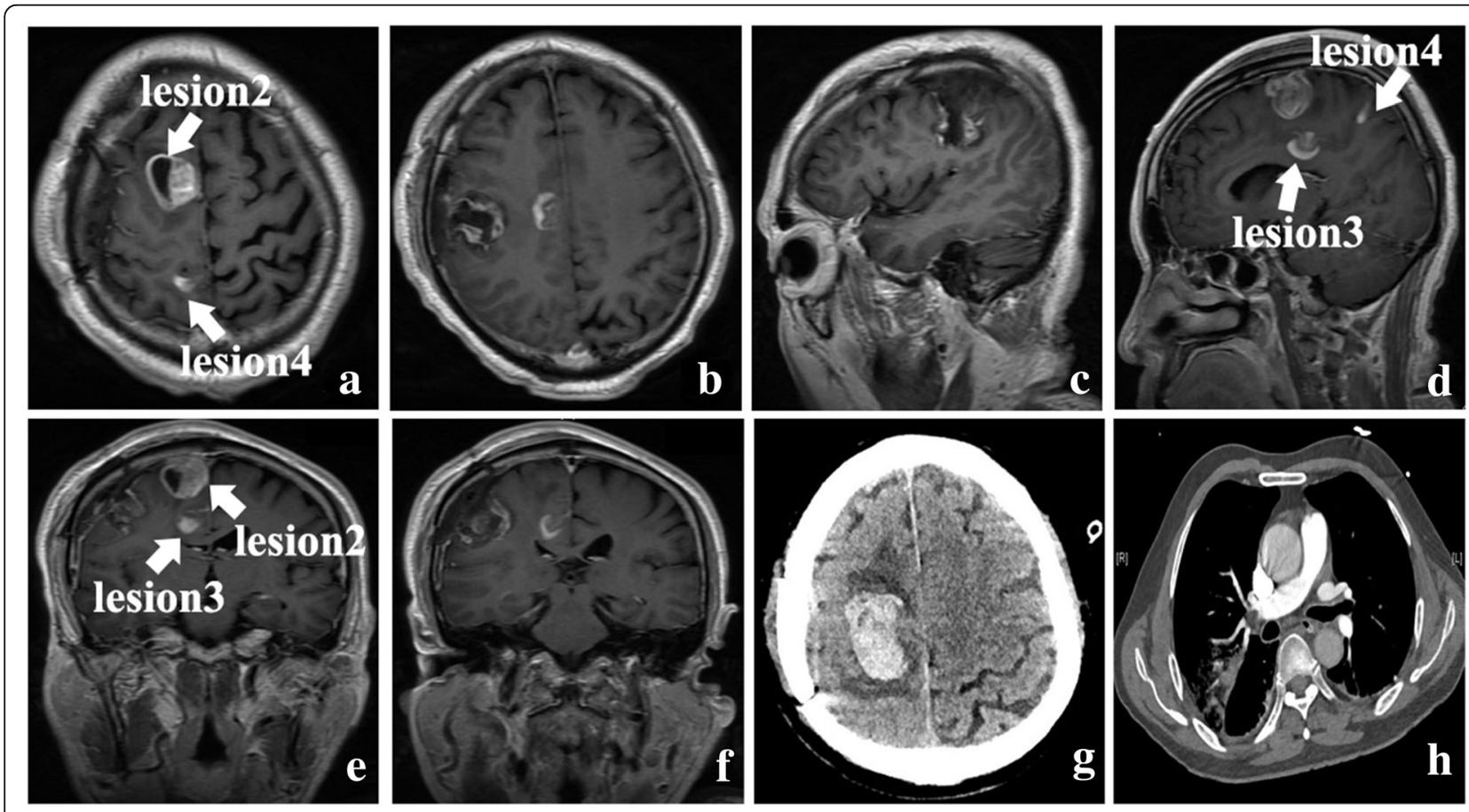

Fig. 5 The axial, sagittal, and coronal contrast-enhanced MRI images after surgery (a-f). Emergent CT scan showed hemorrhage of the residual tumor. $\mathbf{g}$ The CT angiography of pulmonary artery showed a sign of pulmonary artery embolism (h) 
entities or brain abcesses $[14,15]$. In our cases, contrast enhancement MRI played a role in detecting and assessing these lesions, contributing to the surgical resection safely, and PET-CT was advantageous to discern the primary malignancies in other tissues of body accurately and safely by providing detailed functional and metabolic molecular information. We hold the opinion that it is inevitable to perform the PET-CT to distinguish these lesions under this circumstance; besides, infectious and inflammatory indicators should be taken into consideration to exclude other diseases. Multiple lesions bring great difficulties and risks to surgeons so they are always faced with tough choices. It was uncommon to achieve resection of multiple lesions and $70 \%$ of patients underwent resection of only one lesion [9]; thus, tumors tend to invade and relapse sequentially. Recent studies recommend resection of multiple lesions synchronously. Hassaneen et al. reported that aggressive resection of all lesions resulted in a survival duration comparable with that of patients undergoing resection for a single lesion, without an associated increase in postoperative morbidity [12]; thus, the resection of multiple lesions may be a beneficial prognostic factor in these patients. In our case 1 , we successfully achieved the resection of all lesions, reducing the tumor burden as much as possible; though higher risks are posed potentially, postoperative complications did not occur either. In our case 2, the patient underwent the resection of a single lesion located in the right parietal lobe; nevertheless, hemorrhage of residual tumor occurred postoperatively. Early detection and treatment of this complication can contribute to improve the clinical outcomes; even removal of intracranial hematoma surgically will be beneficial if necessary. Regrettably, the pulmonary embolism resulting from coagulopathy occurred and made the treatment contradictorily; eventually, the patient died without a chance of re-operation and additional therapy.

Local irradiation therapies like IMRT and three dimensional conformal RT (3DCRT), which cover the gross target volume locally, now remain the standard adjuvant treatments of unifocal glioblastoma due to their lower toxicity and accuracy. In our case 1 , we adopted IMRT with a dosage of 60.0 Gy in 30 fractions, and sideeffects were not detected during the follow-up period. Recent studies also investigated the safety and efficacy of whole-brain radiotherapy (WBRT) in GBM patients. Lahmi et al. reported their retrospectively study of patients received the WBRT with TMZ and confirmed the safety and efficacy of WBRT, but larger prospective studies are needed to support their results [16]. The Stupp protocol of chemotherapy is widely recommended for newly diagnosed GBM [17]. Liu et al. reported that long-term temozolomide (TMZ) might be an optimal choice for patient with multifocal glioblastoma [7]. In our case 1 , the patient received 2 cycles of sequential TMZ chemotherapy after the concurrent radiochemotherapy, although the general condition of patient remained stable, new lesions emerged, and this might result from the more aggressive phenotype of this disease.

Underlying genetic characteristics of multifocal glioblastoma have not been elucidated clearly. Liu et al. found that multifocal glioblastomas had no IDH1, ATRX, or PDGFRA mutations compared with solitary glioblastomas and were significantly associated with the mesenchymal subtype [8]; Abou-El-Ardat et al. investigated its comprehensive molecular characterization and they found the high frequency of alterations in the 3 GBM core pathways: RTK/PI3K, p53, and RB regulatory pathways, which resemble primary GBMs, suggesting that multifocal glioblastomas develop through parallel genetic evolution [5]. Besides, discordance of IDH mutational status between lesions was also founded in multifocal glioblastoma, indicating the higher heterogeneity compared with unifocal GBM [18]. In our study, although genetic analysis of lesions revealed the distinctive genetic and molecular characteristics of multifocal glioblastoma, more specimens from different patients are needed for evaluating and elucidating their heterogeneity in the future.

Currently, there are still no uniform treatment guidelines for multifocal glioblastoma; thus, further investigation and evaluation are required for unveiling this rare malignancy.

\section{Conclusions}

In summary, we have reported two cases of typical multifocal glioblastoma. The resection of multiple lesions and canonical radio-chemotherapy probably bring survival benefits; meanwhile, further investigation is required for understanding the behavior, management, and outcome of this rare malignancy.

\section{Abbreviations \\ GBM: Glioblastoma multiforme; CT: Computed tomography; PET-CT: Positron emission tomography-computed tomography; MRI: Magnetic resonance imaging; 3DCRT: Three dimensional conformal RT; WBRT: Whole-brain radiotherapy; IMRT: Intensity-modulated radiotherapy; TMZ: Temozolomide}

\section{Acknowledgements}

Not applicable.

\section{Authors' contributions}

Shengqing Lv, Yan Xiang, Xun Qin, Lin Yang, and Zuoxin Zhang participated in the surgery. Ju-Xiang Chen and Bao-Zhong Shi gave us valuable advice on patients' treatment. Guanghui Li helped us in designing the radiochemotherapy. Yao Li conducted a follow-up survey of the patient. Zuoxin Zhang collected the imaging materials and wrote the manuscript. All of the authors critically participated in the manuscript revision. The author(s) read and approved the final manuscript.

\section{Funding}

This work was partly supported by grants from the National Natural Science Foundation of China (NSFC 81972360) and the Joint Research Foundation 
from Chongqing Science and Technology Bureau and Chongqing Municipal Health Commission (2018ZDXM011).

\section{Availability of data and materials}

Not applicable.

\section{Ethics approval and consent to participate}

Not applicable.

\section{Consent for publication}

Written informed consent for publication of patients' individual details and images were obtained from the patients' families.

\section{Competing interests}

The authors declare that they have no competing interests.

\section{Author details}

'Department of Neurosurgery, Xinqiao Hospital, Third Military Medical University, No.183 Xinqiao Street, Shapingba District, Chongqing City 400037, People's Republic of China. ${ }^{2}$ Department of Neurosurgery, Changzheng Hospital and Shanghai Institute of Neurosurgery, Second Military Medical University, Shanghai 200003, People's Republic of China. ${ }^{3}$ Department of Critical Care Medicine \& Department of Neurosurgery, The First Affiliated Hospital, College of Clinical Medicine of Henan University of Science and Technology, Luoyang 471003, Henan, People's Republic of China. ${ }^{4}$ Institute for Cancer Research in People's Liberation Army, Xinqiao Hospital, Third Military Medical University, Chongqing 400037, People's Republic of China.

Received: 24 April 2020 Accepted: 14 December 2020

Published online: 15 January 2021

\section{References}

1. Tang W, Wang D, Shao L, Liu X, Zheng J, Xue Y, et al. LINC00680 and TTNAS1 stabilized by EIF4A3 promoted malignant biological behaviors of glioblastoma cells. Mol Ther Nucleic Acids. 2020;19:905-21.

2. Zhang H, Wang R, Yu Y, Liu J, Luo T, Fan F. Glioblastoma treatment modalities besides surgery. J Cancer. 2019;10(20):4793-806.

3. Rotin DL, Paklina OV, Kobiakov GL, Shishkina LV, Kravchenko ÉV, Stepanian MA. Lung cancer metastases to the brain: clinical and morphological prognostic factors. Zh Vopr Neirokhir Im N N Burdenko. 2013;77(1):24-8.

4. Rick JW, Shahin M, Chandra A, Dalle Ore C, Yue JK, Nguyen A, et al. Systemic therapy for brain metastases. Crit Rev Oncol Hematol. 2019; 142:44-50.

5. Abou-El-Ardat K, Seifert M, Becker K, Eisenreich S, Lehmann M Hackmann K, et al. Comprehensive molecular characterization of multifocal glioblastoma proves its monoclonal origin and reveals novel insights into clonal evolution and heterogeneity of glioblastomas. Neuro Oncol. 2017:19(4):546-57.

6. Paulsson AK, Holmes JA, Peiffer AM, Miller LD, Liu W, Xu J, et al. Comparison of clinical outcomes and genomic characteristics of single focus and multifocal glioblastoma. J Neurooncol. 2014;119(2):429-35.

7. Liu Y, Hao S, Yu L, Gao Z. Long-term temozolomide might be an optimal choice for patient with multifocal glioblastoma, especially with deep-seated structure involvement: a case report and literature review. World J Surg Oncol. 2015:13:142

8. Liu Q, Liu Y, Li W, Wang X, Sawaya R, Lang FF, et al. Genetic, epigenetic, and molecular landscapes of multifocal and multicentric glioblastoma. Acta Neuropathol. 2015;130(4):587-97.

9. Di Carlo DT, Cagnazzo F, Benedetto N, Morganti R, Perrini P. Multiple highgrade gliomas: epidemiology, management, and outcome. A systematic review and meta-analysis. Neurosurg Rev. 2019;42(2):263-75.

10. Barnard RO, Geddes JF. The incidence of multifocal cerebral gliomas. A histologic study of large hemisphere sections. Cancer. 1987;60(7):1519-31.

11. Hassaneen W, Levine NB, Suki D, Salaskar AL, de Moura LA, McCutcheon IE, et al. Multiple craniotomies in the management of multifocal and multicentric glioblastoma. Clinical article. J Neurosurg. 2011;114(3):576-84.

12. Patil CG, Yi A, Elramsisy A, Hu J, Mukherjee D, Irvin DK, et al. Prognosis of patients with multifocal glioblastoma: a case-control study. J Neurosurg. 2012;117(4):705-11.
13. Lasocki A, Gaillard F, Tacey M, Drummond K, Stuckey S. Multifocal and multicentric glioblastoma: Improved characterisation with FLAIR imaging and prognostic implications. J Clin Neurosci. 2016;31:92-8.

14. Prather JL, Long JM, van Heertum R, Hardman J. Multicentric and isolated multifocal glioblastoma multiforme simulating metastatic disease. $\mathrm{Br} \mathrm{J}$ Radiol. 1975;48(565):10-5.

15. Kyritsis AP, Levin VA, Yung WK, Leeds NE. Imaging patterns of multifocal gliomas. Eur J Radiol. 1993;16(3):163-70.

16. Lahmi L, Idbaih A, Rivin Del Campo E, Hoang-Xuan K, Mokhtari K, Sanson $M$, et al. Whole brain radiotherapy with concurrent temozolomide in multifocal and/or multicentric newly diagnosed glioblastoma. J Clin Neurosci. 2019;68:39-44.

17. Stupp R, Mason WP, van den Bent MJ, Weller M, Fisher B, Taphoorn MJ, et al. Radiotherapy plus concomitant and adjuvant temozolomide for glioblastoma. N Engl J Med. 2005:352(10):987-96.

18. Lombardi G, Della Puppa A, Gardiman MP, Rossi S, Candiotto C, et al. Discordance of IDH mutational status between lesions in an adult patient with multifocal glioma. Neuro Oncol. 2018;20(8):1142-3.
Ready to submit your research? Choose BMC and benefit from:

- fast, convenient online submission

- thorough peer review by experienced researchers in your field

- rapid publication on acceptance

- support for research data, including large and complex data types

- gold Open Access which fosters wider collaboration and increased citations

- maximum visibility for your research: over $100 \mathrm{M}$ website views per year

At BMC, research is always in progress.

Learn more biomedcentral.com/submissions 